\title{
BMJ Open Major determinant of the occurrence of pacing-induced cardiomyopathy in complete atrioventricular block: a multicentre, retrospective analysis over a 15-year period in South Korea
}

Jun Hyung Kim, ${ }^{1}$ Ki-Woon Kang, ${ }^{2}$ Jung Yeon Chin, ${ }^{2}$ Tae-Seok Kim, ${ }^{3}$ Jae-Hyeong Park, ${ }^{1}$ Yu Jeong Choi ${ }^{2}$

To cite: Kim JH, Kang K-W, Chin JY, et al. Major determinant of the occurrence of pacing-induced cardiomyopathy in complete atrioventricular block: a multicentre, retrospective analysis over a 15 -year period in South Korea. BMJ Open 2018;8:e019048. doi:10.1136/ bmjopen-2017-019048

- Prepublication history and additional material for this paper are available online. To view these files, please visit the journal online (http://dx.doi org/10.1136/bmjopen-2017019048).

Received 6 0ctober 2017 Revised 12 December 2017 Accepted 14 December 2017

Check for updates

${ }^{1}$ Division of Cardiology, Chungnam National University Hospital, Daejeon, Korea

${ }^{2}$ Division of Cardiology, Eulji University Hospital, Daejeon, Korea

${ }^{3}$ Division of Cardiology, Catholic Saint Hospital, Daejeon, Korea

Correspondence to Dr Ki-Woon Kang;

kwkang@eulji.ac.kr

\section{ABSTRACT}

Objectives The predictors of pacing-induced cardiomyopathy (PICM) for complete atrioventricular block (CAVB) have not yet been defined. The aim of this study was to investigate the major determinant of the occurrence of PICM.

Setting This is a multicentre, retrospective analysis of CAVB from tertiary referral centres in Daejeon, South Korea.

Participants A cohort of 900 consecutive patients with an implanted pacemaker was collected from December 2001 to August 2015. Of these, a total of 130 patients with CAVB with pacing-dependent rhythm who underwent ECG and echocardiogram before and after implantation were analysed for the occurrence of PICM.

Outcome measures Cox proportional hazards models evaluated the determinant of PICM by ECG, device parameters and echocardiogram over a mean of 4.5 years. Results PICM was observed in $16.1 \%(n=21)$ of all patients with CAVB (age, $64 \pm 11$ years; male, $36.2 \%$ ). The preimplant left ventricular (LV) ejection fraction $(66 \% \pm 9 \%$ vs $66 \% \pm 8 \%$ ) and non-apical pacing ( $40.4 \%$ vs $33.3 \%)$ were similar; however, the native QRS duration (124 \pm 34 $\mathrm{ms}$ vs $149 \pm 32 \mathrm{~ms}$ ) and the paced QRS duration (pQRSd) (139 $\pm 29 \mathrm{~ms}$ vs $167 \pm 28 \mathrm{~ms}$ ) were significantly different between the two groups. The postimplant LV ejection fraction $(61 \% \pm 7 \%$ vs $31 \% \pm 8 \%)$ was also significantly different at the end of follow-up. A pQRSd significantly correlated with PICM (HR 1.05, 95\% Cl 1.02 to 1.09 , $\mathrm{P}=0.001)$. A pQRSd with a cut-off value of above $140 \mathrm{~ms}$ had a sensitivity of $95 \%$ while a pQRSd with a cut-off value of above $167 \mathrm{~ms}$ had a specificity of $90 \%$ for PICM. Conclusion In patients with CAVB with pacing-dependent rhythm, regardless of the pacing site, the $\mathrm{PQRSd}$ is a major determinant of the occurrence of PICM.

\section{INTRODUCTION}

Pacemakers have been a definite treatment tool for symptomatic bradyarrhythmia to reduce cardiac morbidity and mortality. ${ }^{1}$ However, chronic right ventricular (RV) pacing has a potentially deleterious effect on
Strengths and limitations of this study

- This is a multicentre, retrospective data analysis of complete atrioventricular block over a 15-year period.

- This study included relatively small-sized patients in three referral centres in South Korea and may limit the generalisation of the results.

left ventricular (LV) function. ${ }^{1-4}$ This deleterious effect of chronic RV pacing on LV function is known as pacing-induced cardiomyopathy (PICM). ${ }^{14-6}$ Several studies have demonstrated that pacing anatomical site, pacing burden and preimplant LV dysfunction affect the occurrence of PICM and its subsequent clinical outcomes. ${ }^{13}$ 7-9 In particular, recognition of predictors for the occurrence of PICM may lead to better identification of patients at high risk for complete atrioventricular block (CAVB) with pacing-dependent rhythm. However, because PICM does not occur in all patients with CAVB with chronic RV pacing, timely and proper evaluation should be considered for those who most likely develop PICM. ${ }^{7}$ Therefore, we retrospectively analysed a large cohort to identify the major determinants of the occurrence of PICM in patients with CAVB with pacing-dependent rhythm over a long period of time.

\section{METHOD}

Study population

Consecutive patients with an implanted pacemaker were retrospectively collected from three different tertiary referral centres-Eulji University Hospital, Chungnam National University and Catholic St Mary's Hospital, which are located in Daejeon, South 
Korea-from December 2001 to August 2015. Among a total of 900 patients with an implanted pacemaker, patients with sick sinus syndrome, paroxysmal and advanced atrioventricular (AV) block $(\mathrm{n}=482)$, persistent/permanent atrial fibrillation (AF) $(\mathrm{n}=140)$ and preimplant LV dysfunction $(\mathrm{n}=148)$ combined with ischaemic heart disease, ${ }^{10}$ including acute coronary syndrome, other proven cardiomyopathy or severe valvular disease at the preimplant period, were excluded from the study. Our investigators excluded pre-existing persistent/permanent $\mathrm{AF}$ which are considered risk factors for the occurrence of heart failure and could influence the relationship between CAVB and PICM. Thus, patients with CAVB $(n=130)$ with documented preimplant and postimplant LV ejection fraction (LVEF) were analysed in this study (see online supplementary figure 1). All patients provided informed consent, and the study protocol conforms to the ethical guidelines of the 1975 Declaration of Helsinki.

LVEF was measured at Eulji University Hospital, Chungnam National University Hospital and St Mary's Hospital using standard echocardiographic techniques. Preimplant and postimplant (at least 1 day after the index implant) echocardiograms were performed and interpreted by two experienced cardiologists who were echocardiogram specialists (JY Chin at Eulji University Hospital and J-H Park at Chungnam National University Hospital). None of the patients developed myocardial infarction during the follow-up period, and the baseline clinical and demographic data, ECG and echocardiogram, and medication data were acquired from the electronic medical records.

\section{ECG parameters, pacemaker data and definition of PICM}

Baseline ECG parameters were acquired from the ECG that was performed closest to the implant period using the standard criteria established by the American Heart Association and Heart Rhythm Society Expert Consensus. ${ }^{11}$ RV pacing-leads sites were reviewed using the standard X-ray (see online supplementary figure 2). Pacemaker data were also acquired at regular intervals (at least 6 months), and the pacing burden (atrial and ventricular pacing \%) was recorded at the time of follow-up and PICM diagnosis. Native QRS duration (nQRSd) was measured within 7 days at preimplant state, and paced QRS duration (pQRSd) was measured also within 7 days at the postimplant state from the surface 12-lead ECG.

PICM was defined as greater than a $10 \%$ decrease in LVEF, with a resultant LVEF less than $50 \%$, as previously reported, ${ }^{7}$ regardless of heart failure symptoms ${ }^{412} 13$ (see online supplementary videos 1 and 2). The time of PICM occurrence was considered the date of the first decrease in LVEF determined by echocardiogram with documented ECG at the time during the follow-up period.

\section{Statistical analysis}

Baseline clinical, ECG, echocardiogram and pacemaker interrogation data of the enrolled patients were compared between those without PICM and with PICM using independent $\mathrm{t}$-test and $\mathrm{X}^{2}$ test. To determine the independent predictors of PICM occurrence, the multivariate Cox regression hazard model was used for PICM. A receiver operating characteristic (ROC) curve was plotted to identify the cut-off value with the best sensitivity and specificity for the occurrence of PICM, and a Kaplan-Meier curve was plotted for free-from-PICM survival. Analyses were performed with the MedCalc software (V.17.0, Ostend, Belgium). P values $<0.05$ were considered statistically significant.

\section{RESULTS}

Comparison of baseline characteristics between patients with and without PICM

Among all patients, 130 patients with CAVB with implanted pacemakers (dual-chamber: $84.6 \%$ ) were suitable for the analysis of PICM in this study. The average age $(64 \pm 11$ years vs $62 \pm 11$ years), the proportion of male $(36.7 \%$ vs $33.3 \%)$ and the occurrence of $\mathrm{AF}(14.6 \%$ vs $14.2 \%$ ) were detected among patients without PICM and with PICM during the follow-up period, and the mean duration of follow-up (4.8 \pm 3.5 years vs $4.2 \pm 3.5$ years) was similar between patients without PICM and with PICM. Other baseline clinical characteristics, except for diabetes and previous stroke, were also similar between patients without PICM and with PICM. Among the laboratory data, haemoglobin and total bilirubin levels, which are associated with heart failure, were similar between patients without PICM and with PICM at preimplant and postimplant stages (table 1 ).

\section{Comparison of ECG data between patients with and without PICM}

Among the 130 patients, 109 maintained normal LV function until the end of follow-up. The remainder of patients with CAVB $(\mathrm{n}=21,16.1 \%)$ were considered to have PICM, with a decrease in LVEF from $65 \% \pm 10 \%$ at baseline to $37 \% \pm 8 \%$. The follow-up ventricular pacing burden was similar between patients without PICM and with PICM $(85 \% \pm 18 \%$ vs $85 \% \pm 17 \%)$. Compared with the patients without PICM, the patients who developed PICM had a significantly wider nQRSd $(124 \pm 34 \mathrm{~ms}$ vs $149 \pm 32$ $\mathrm{ms}, \mathrm{P}=0.004)$, QTc interval $(466 \pm 54 \mathrm{~ms}$ vs $495 \pm 44 \mathrm{~ms}$, $\mathrm{P}=0.035)$ and $\mathrm{pQRSd}(139 \pm 29 \mathrm{~ms}$ vs $167 \pm 28 \mathrm{~ms}, \mathrm{P}<0.001)$ (table 2).

\section{Comparison of medications between patients with and without PICM}

Unlike patients without PICM, patients with PICM more frequently took ACE inhibitor or angiotensin II receptor blocker medication before implantation, and betablockers and diuretics after implantation, as shown in table 3 .

\section{Predictors of PICM occurrence}

Multivariate Cox regression analysis showed that nQRSd had an HR of 1.01 and a 95\% CI of 1.00 to 1.03 with a P 
Table 1 Baseline characteristics between patients with and without pacing-induced cardiomyopathy (PICM)

\begin{tabular}{|c|c|c|c|c|}
\hline & $\begin{array}{l}\text { All } \\
\text { patients } \\
\end{array}$ & $\begin{array}{l}\text { Without } \\
\text { PICM }\end{array}$ & $\begin{array}{l}\text { With } \\
\text { PICM }\end{array}$ & \\
\hline & $n=130$ & $n=109$ & $\mathrm{n}=\mathbf{2 1}$ & P value \\
\hline Age, years & $64 \pm 11$ & $64 \pm 11$ & $62 \pm 11$ & 0.472 \\
\hline Male, n (\%) & 47 (36.2) & $40(36.7)$ & 7 (33.3) & 0.768 \\
\hline $\begin{array}{l}\text { Hypertension, } \\
\text { n (\%) }\end{array}$ & $75(57.7)$ & $58(53.2)$ & $16(76.2)$ & 0.146 \\
\hline Diabetes, n (\%) & $31(24.0)$ & $30(27.5)$ & $1(5.0)$ & $0.030^{*}$ \\
\hline $\begin{array}{l}\text { Ischaemic } \\
\text { heart disease, } \\
\text { n (\%) }\end{array}$ & 15 (11.5) & $11(10.1)$ & 4 (19.0) & 0.239 \\
\hline $\begin{array}{l}\text { Stroke or } \\
\text { transient } \\
\text { ischaemic } \\
\text { attack, n (\%) }\end{array}$ & $9(6.9)$ & $5(4.6)$ & $4(19.0)$ & $0.017^{*}$ \\
\hline Alcohol, n (\%) & $16(12.3)$ & $13(11.9)$ & $3(13.3)$ & 0.763 \\
\hline Smoking, n (\%) & $18(13.8)$ & $16(14.7)$ & $2(9.5)$ & 0.531 \\
\hline $\begin{array}{l}\text { Haemoglobin, } \\
\text { g/L }\end{array}$ & $12.3 \pm 2.1$ & $12.1 \pm 1.9$ & $12.4 \pm 2.6$ & 0.660 \\
\hline $\begin{array}{l}\text { Total bilirubin, } \\
\mathrm{mg} / \mathrm{dL}\end{array}$ & $1.0 \pm 0.8$ & $1.0 \pm 0.6$ & $1.0 \pm 0.8$ & 0.556 \\
\hline
\end{tabular}

*Statistically significant.

value of 0.051 , and that $\mathrm{pQRSd}$ had an $\mathrm{HR}$ of 1.05 and a $95 \%$ CI of 1.02 to 1.09 with a $\mathrm{P}$ value of $<0.001$ (table 4 ). ROC curve analysis showed that a pQRSd above $140 \mathrm{~ms}$ had the combined best sensitivity (95\%) and specificity $(36 \%)$ and pQRSd above $167 \mathrm{~ms}$ had the combined sensitivity (52\%) and best specificity (90\%) for predicting the occurrence of PICM, with statistical significance (figure 1). In the Kaplan-Meier curve, both pQRSd of $140 \mathrm{~ms}$ and $167 \mathrm{~ms}$ were significantly associated with the occurrence of PICM (log-rank, $\mathrm{P}=0.03$ vs $\mathrm{P}<0.001$; figure 2).

\section{DISCUSSION}

In our study, among patients with CAVB with normal LV function at the preimplant period, PICM occurred in $16.1 \%$ of patients with pacing-dependent rhythm over a mean follow-up duration of $4.7 \pm 3.5$ years. A pQRSd was significantly associated with the occurrence of PICM. In particular, a pQRSd wider than $140 \mathrm{~ms}$ had a sensitivity of 95\% and a pQRSd of $167 \mathrm{~ms}$ had a specificity of $90 \%$ for predicting the occurrence of PICM.

Our result on the incidence of PICM over a long-term follow-up period is comparable with that from previous reports, ranging from $9 \%$ to $26 \%$ depending on the population investigated and the length of follow-up. ${ }^{4}$ We also defined PICM as greater than a $10 \%$ decrease in LVEF, with a resultant LVEF less than $50 \%$ after the index implant. The time to the diagnosis of PICM was defined as the period from the date of implantation to the date of the first documented decrease in LVEF.

PICM has been widely considered as the pacing-associated heart failure. ${ }^{17}$ The significance of PICM has been established for an increased risk in $\mathrm{AF}$, heart failure hospitalisation and cardiac mortality. ${ }^{9}$ The pacing site, increased pacing burden, preimplant LV dysfunction and QRS duration have been considered the independent predictors of PICM. ${ }^{1-4} 714$

First, with regard to the pacing site, a recent meta-analysis has suggested that the LVEF is higher in patients with RV non-apical pacing than those with RV apical pacing. However, this conclusion is still debated due to conflicting results. ${ }^{3}{ }^{15}$ In the PROTECT-PACE study, among patients with a high-grade AV block and preserved LV function, RV non-apical pacing did not have a protective effect on LV function compared with RV apical pacing over a 2-year period. ${ }^{8}$ In addition, Chan $e t \mathrm{al}^{16}$ have previously reported that LV volumes and systolic function after long-term RV pacing could be predicted by $\mathrm{pQRSd}$, but not pacing site. Our multicentre study also showed no significant difference between RV apical and non-apical pacing in the occurrence of PICM (40.4\% vs $33.3 \%, \mathrm{P}=0.546$ ) among patients with CAVB with pacing-dependent rhythm over a long-term follow-up period.

Second, pacing burden has been considered a better predictor for the occurrence of PICM, and previous studies have shown heterogeneous percentages of pacing burden. ${ }^{2}$ In our study, in patients with CAVB who required a high burden of permanent pacing, the confounding factor was minimised using homogeneous percentages of $\mathrm{RV}$ pacing ( $85 \%$ vs $85 \%, \mathrm{P}=0.860$ ) when analysing the predictors of PICM.

Third, with regard to preimplant LV dysfunction, previous studies had baseline pre-existing heart failure associated with coronary artery disease and $\mathrm{AF}^{4}{ }^{7}$ and in the PREDICT-HF trial pre-existing heart failure was highly associated with pQRSd. Other studies have also found $\mathrm{pQRSd}$ to be an important predictor of heart failure among patients with chronic RV pacing. ${ }^{13} 1718$ In our study, all patients with CAVB with pre-existing LV systolic dysfunction (with or without heart failure) were excluded, and our results were reliable enough to include the analysis of PICM compared with previous studies.

Fourth, pacing-induced electrical dyssynchrony developed mechanical dyssynchrony; thus, the pQRSd could be a strong and independent determinant of the occurrence of PICM. ${ }^{6}$ Our data also show that the pQRSd related to LV mechanical dyssynchrony has been confirmed to be significantly associated with LV remodelling (representative of the dyssynchrony index with strain in the two-dimensional or three-dimensional parameters; online supplementary videos 3 and 4), resulting in the occurrence of PICM by echocardiogram.

Pap et $a l^{19}$ reported that nQRSd could be positively correlated with $\mathrm{pQRSd}$, although the nQRSd as escape rhythm is influenced by the level of antegrade block on the His-Purkinje system during AV block. In addition, an 
Table 2 Comparison of ECG parameters between patients with and without PICM

\begin{tabular}{|c|c|c|c|c|}
\hline & All patients & Without PICM & With PICM & \multirow[b]{2}{*}{$P$ value } \\
\hline & $n=130$ & $n=109$ & $\mathrm{n}=21$ & \\
\hline \multicolumn{5}{|l|}{ Preimplant } \\
\hline Ejection fraction, n (\%) & $65 \pm 10$ & $66 \pm 9$ & $65 \pm 10$ & 0.607 \\
\hline Left atrial diameter, $\mathrm{mm}$ & $39 \pm 9$ & $38 \pm 7$ & $40 \pm 8$ & 0.552 \\
\hline Heart rate, bpm & $60 \pm 30$ & $57 \pm 18$ & $60 \pm 12$ & 0.550 \\
\hline PR interval, ms & $190 \pm 81$ & $170 \pm 115$ & $213 \pm 130$ & 0.203 \\
\hline QRS duration, ms & $136 \pm 26$ & $124 \pm 34$ & $149 \pm 32$ & $0.004^{*}$ \\
\hline QTc interval, ms & $480 \pm 37$ & $466 \pm 54$ & $495 \pm 44$ & $0.035^{\star}$ \\
\hline \multicolumn{5}{|l|}{ Postimplant } \\
\hline Dual-chamber, n (\%) & $110(84.6)$ & 90 (82.5) & $20(95.2)$ & 0.142 \\
\hline Ejection fraction, n (\%) & $45 \pm 8$ & $61 \pm 7$ & $37 \pm 8$ & $<0.001^{*}$ \\
\hline Left atrial diameter, $\mathrm{mm}$ & $40 \pm 7$ & $39 \pm 7$ & $40 \pm 6$ & 0.266 \\
\hline $\begin{array}{l}\text { Occurrence of atrial fibrillation, } \mathrm{n} \\
(\%)\end{array}$ & 19 (14.6) & $16(14.6)$ & $3(14.2)$ & 0.962 \\
\hline Heart rate, bpm & $68 \pm 30$ & $69 \pm 14$ & $67 \pm 9$ & 0.616 \\
\hline PR interval, ms & $178 \pm 81$ & $168 \pm 80$ & $187 \pm 62$ & 0.337 \\
\hline Paced QRS duration, ms & $149 \pm 26$ & $139 \pm 29$ & $167 \pm 28$ & $<0.001^{*}$ \\
\hline Paced QRS axis, degree & $2 \pm 78$ & $2 \pm 78$ & $1 \pm 91$ & 0.971 \\
\hline Paced QTc interval, ms & $490 \pm 37$ & $484 \pm 46$ & $496 \pm 36$ & 0.254 \\
\hline Non-apical pacing, \% & $51(39.1)$ & $44(40.4)$ & $7(33.3)$ & 0.546 \\
\hline Atrial pacing, $\%$ & $23 \pm 22$ & $23 \pm 23$ & $22 \pm 22$ & 0.954 \\
\hline Ventricular pacing, \% & $85 \pm 17$ & $85 \pm 18$ & $85 \pm 17$ & 0.860 \\
\hline
\end{tabular}

*Statistically significant.

bpm, beats per minute; PICM, pacing-induced cardiomyopathy.

nQRSd above 115 ms was highly specific (90\%) for the occurrence of PICM, as reported in a single-centre study. ${ }^{20}$ A single-centre study by Khurshid et $a l^{13}$ also suggested that the nQRSd ( $\mathrm{HR}=1.03$ per ms; $\mathrm{P}<0.001)$ is an independent predictor of PICM occurrence. In comparison, our study demonstrated that the proportion of patients with an nQRSd above $115 \mathrm{~ms}$ is higher in patients with PICM than those without PICM (74\% vs $55 \%)$. In particular, the $\mathrm{nQRSd}(\mathrm{HR}=1.02 ; \mathrm{P}=0.010)$ was slightly significant in our univariate analysis and exhibited a positive trend $(\mathrm{HR}=1.01 ; \mathrm{P}=0.051)$ in the multivariate analysis of the occurrence of PICM (table 4). It is implicated that a

Table 3 Comparison of medications between patients with and without PICM

\begin{tabular}{|c|c|c|c|c|}
\hline & \multirow{2}{*}{$\begin{array}{l}\text { All patients } \\
n=130\end{array}$} & \multirow{2}{*}{$\begin{array}{l}\text { Without PICM } \\
n=109\end{array}$} & \multirow{2}{*}{$\begin{array}{l}\text { With PICM } \\
n=21\end{array}$} & \multirow[b]{2}{*}{$P$ value } \\
\hline & & & & \\
\hline \multicolumn{5}{|l|}{ Preimplant } \\
\hline Beta-blocker, n (\%) & $16(12.3)$ & $11(10.1)$ & $5(23.8)$ & 0.080 \\
\hline CCB, n (\%) & $26(20.0)$ & $22(20.2)$ & 4 (19.0) & 0.905 \\
\hline \multicolumn{5}{|l|}{ Postimplant } \\
\hline ACEI or ARB, n (\%) & $58(44.6)$ & $45(41.3)$ & $13(61.9)$ & 0.082 \\
\hline Beta-blocker, n (\%) & $22(16.9)$ & $15(13.8)$ & 7 (33.8) & $0.029^{\star}$ \\
\hline CCB, n (\%) & $31(23.8)$ & $28(25.7)$ & $3(14.3)$ & 0.262 \\
\hline Diuretics, n (\%) & $32(24.6)$ & $23(21.1)$ & $9(42.9)$ & $0.034^{\star}$ \\
\hline
\end{tabular}

*Statistically significant.

ACEI, ACE inhibitor; ARB, angiotensin receptor blocker; CCB, calcium channel blocker; PICM, pacing-induced cardiomyopathy. 
Table 4 Cox regression analysis for the occurrence of pacing-induced cardiomyopathy

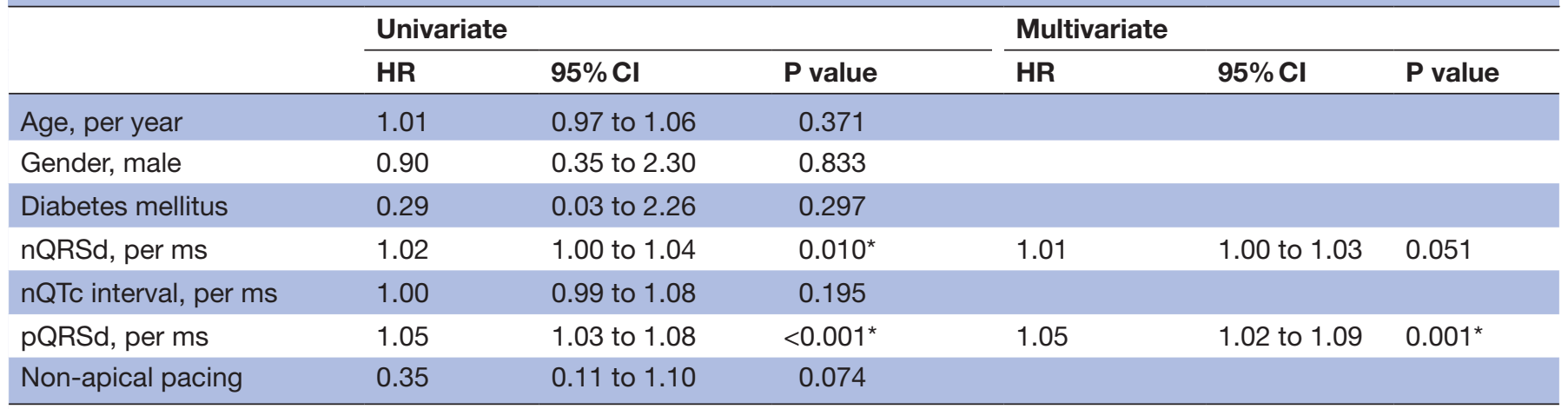

*Statistically significant.

nQRSd, native QRS duration; $\mathrm{pQRSd}$, paced QRS duration.

wider $\mathrm{nQRSd}$ may be a predisposition to cardiomyopathy. In particular, among patients with CAVB with normal LV function before implant, wider $\mathrm{nQRSd}$ may reflect more pathological electrical His-Purkinje conduction.

Miyoshi et $a l^{21}$ also proposed that a pQRSd wider than $190 \mathrm{~ms}$ suggested a higher rate of morbidity than a pQRSd below $190 \mathrm{~ms}$. However, the enrolled patients had ischaemic heart failure, valvular heart disease and other causes of cardiomyopathy, whereas our study did not. Chen et al ${ }^{17}$ prospectively showed in 194 patients with CAVB without heart failure over a 3-year follow-up that clinical heart failure events were higher and the LVEF was lower among patients with a wider pQRSd. In addition, a pQRSd of $165 \mathrm{~ms}$ had the best specificity $(67 \%)$ for predicting heart failure, and a single-centre study by

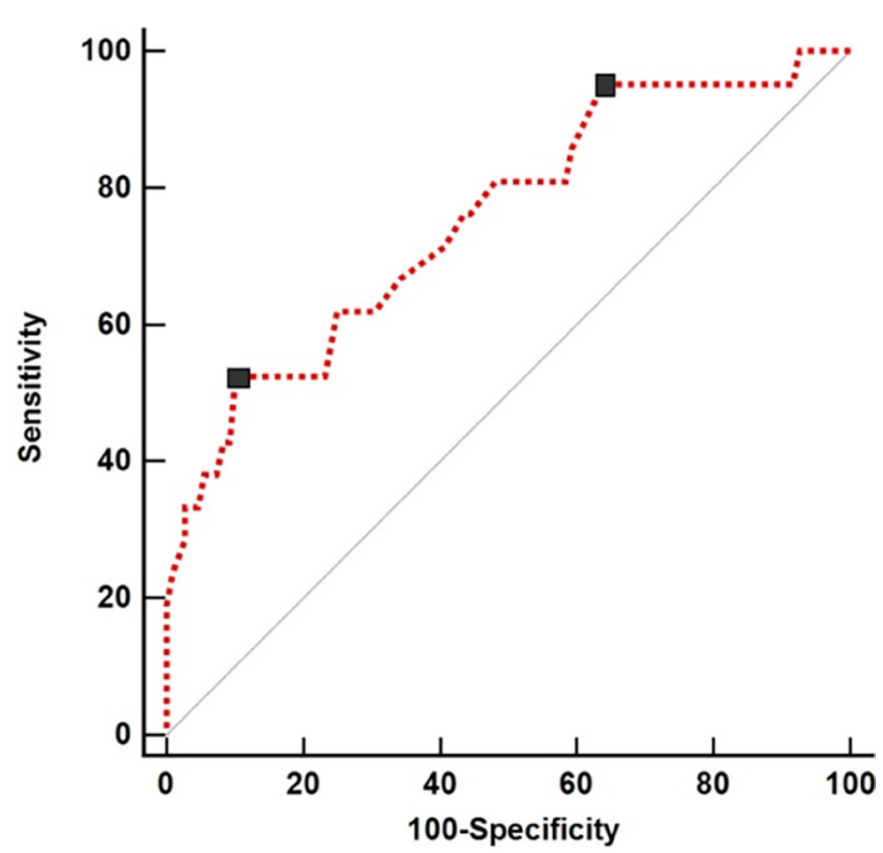

Figure 1 Receiver operating characteristic curve analysis showing the pQRSd had correlated with the occurrence of pacing-induced cardiomyopathy and two rectangular black marks showing the best sensitivity ( $p Q R S d$ $140 \mathrm{~ms}$ ) and specificity (pQRSd $167 \mathrm{~ms}$ ) with statistical significance. $\mathrm{pQRSd}$, paced QRS duration.
Khurshid et $a l^{13}$ also proposed that a pQRSd of $150 \mathrm{~ms}$ was a sensitive marker for PICM; however, those enrolled patients also had pre-existing AF, coronary artery disease and unknown cardiomyopathies.
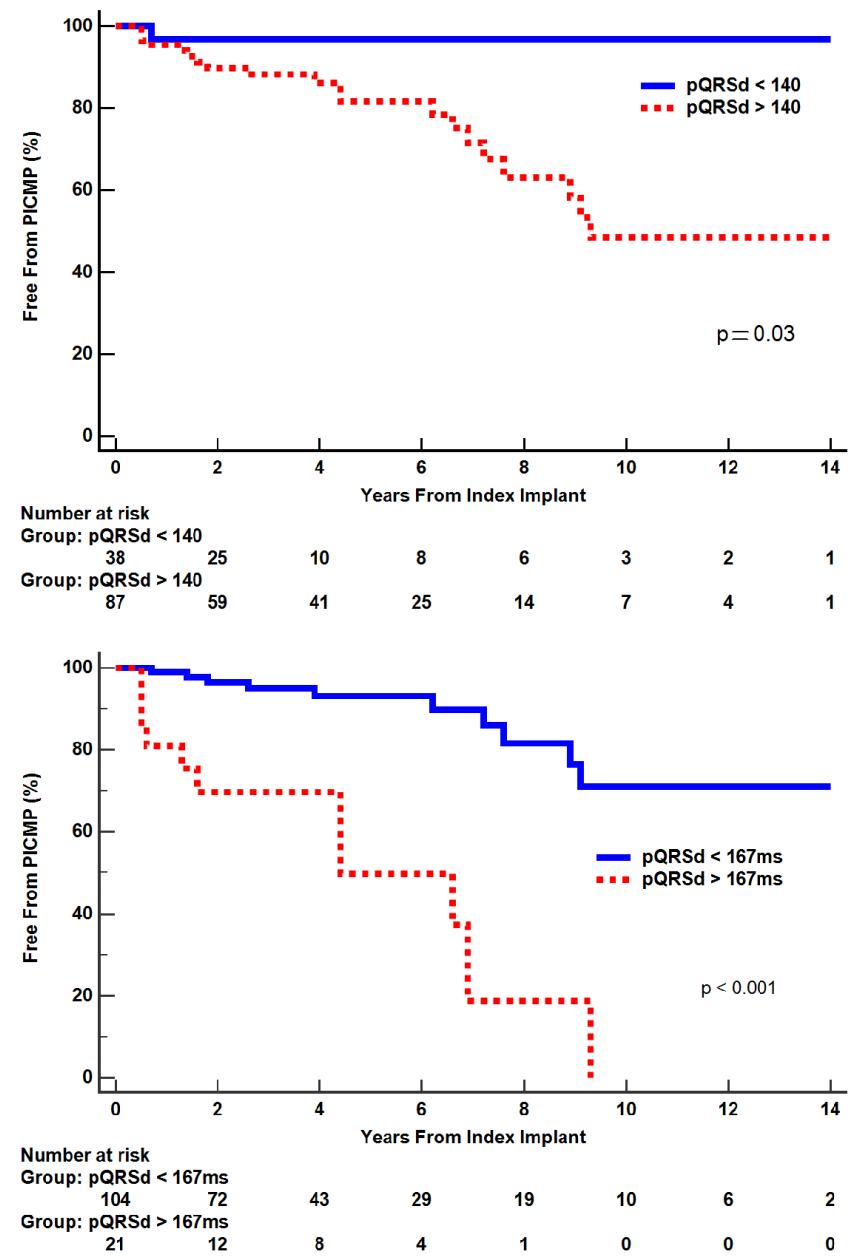

Figure 2 Kaplan-Meier curve analysis showing free-fromPICM survival with a pQRSd (cut-off value of $140 \mathrm{~ms}$ and 167 ms). PICM, pacing-induced cardiomyopathy; pQRSd, paced QRS duration. 
Taken together, a wider $\mathrm{pQRSd}$ could be a major determinant of the occurrence of PICM. We also found that delayed signs and symptoms of heart failure reduce the early detection of PICM in patients with pacing-dependent rhythm and that not all patients with PICM meet the clinical criteria for heart failure despite a significant reduction of LVEF. This is consistent with previous studies showing only low sensitivity for the diagnosis of heart failure with reduced LV function. ${ }^{22}$ Therefore, a more sensitive and specific marker for PICM occurrence may be required for patients with pacing-dependent rhythm.

Our study analysed a contemporary cohort of patients with CAVB and provided a detailed characterisation of the clinical, electrocardiographic, laboratory and echocardiographic data at both preimplant and postimplant periods, as well as at the end of the follow-up. In particular, it could be noteworthy that a multicentre study with a longer follow-up duration distinguish it from previous studies, as well as complement the previous studies. ${ }^{13} 14$

Patients with PICM mostly showed a prolonged pQRSd $>140$ ms while those without PICM rarely show a prolonged $\mathrm{pQRSd}>167 \mathrm{~ms}$. Therefore, even though pQRSd correlates with the occurrence of PICM, pQRSd $<140 \mathrm{~ms}$ could exclude the occurrence of PICM and $\mathrm{pQRSd}>167 \mathrm{~ms}$ could not exclude non-PICM state for follow-up.

Our findings suggest that patients with a wider $\mathrm{pQRSd}$ are at higher risk for developing PICM, and therefore these patients may benefit from routine echocardiographic screening for PICM and possibly a lower threshold for early biventricular or His-bundle pacing. ${ }^{23} 24$

This study included relatively small number of patients in the three referral centres in South Korea and may limit the generalisation of the results due to several limitations. First, our study was a retrospective study with unmeasured selection bias, and patients without preimplant or postimplant echocardiogram were excluded for analysis. Our study suffers from a small number of patients with PICM due to low incidence of PICM and lack of associations may be raised due to power issues. Second, this was a multicentre study, and thus the influence of different physicians on clinical decision-making may also influence the clinical variables associated with heart failure. Third, the definition of PICM was defined only with LVEF based on anecdotal evidence from a previous study. An appropriate universal definition of PICM is needed..$^{25}$ Fourth, while we excluded all potential aetiologies of heart failure, it is speculated that a wider nQRSd is associated with the occurrence of PICM because it reflects cardiomyopathy with normal LV function at preimplant stage. Thus, more detailed studies on the relationship between the nQRSd and an electrical pathology or substrate in patients with CAVB with normal LV function are needed. Fifth, the ability to upgrade to biventricular pacing or Hi-bundle pacing for a pQRSd over $150 \mathrm{~ms}$ was limited in patients with PICM in our study because of the strict coverage of the national health insurance.
Early detection and preventive management of PICM are challenging in patients with pacing-dependent rhythm because there are few data to guide clinicians in identifying subclinical and clinical PICM in the subsequent months to years after pacemaker implantation.

\section{CONCLUSION}

The occurrence of PICM in patients with pacing-dependent rhythm seems to be common but cannot be reliably diagnosed based on the conventional heart failure criteria. The $\mathrm{pQRSd}$, which was more significant than the nQRSd, is associated with the occurrence of PICM. In particular, a patient with a pQRSd above $140 \mathrm{~ms}$ had the best sensitivity and a pQRSd above $167 \mathrm{~ms}$ had the best specificity of occurrence for PICM. Regardless of the pacing site, the pQRSd should be monitored for the timely evaluation and proper management of patients at high risk of PICM to reduce cardiac morbidity and mortality over a long follow-up period.

Contributors K-W K designed the study and revised the manuscript in the final version. JH K, YJ C and T-S K collected and analysed the clinical characteristics, ECG and pacemaker interrogation data. JY C and J-H P collected and analysed the echocardiogram data.

Funding This study was supported by a grant from the Korean Healthcare Technology R\&D project, which is funded by the Ministry of Health and Welfare (2017R1D1A3B03030919).

Competing interests None declared.

Patient consent Obtained.

Ethics approval The study was approved by the institutional review board of each centre in South Korea.

Provenance and peer review Not commissioned; externally peer reviewed. Data sharing statement No additional data available.

Open Access This is an Open Access article distributed in accordance with the Creative Commons Attribution Non Commercial (CC BY-NC 4.0) license, which permits others to distribute, remix, adapt, build upon this work non-commercially, and license their derivative works on different terms, provided the original work is properly cited and the use is non-commercial. See: http://creativecommons.org/ licenses/by-nc/4.0/

(c) Article author(s) (or their employer(s) unless otherwise stated in the text of the article) 2018. All rights reserved. No commercial use is permitted unless otherwise expressly granted.

\section{REFERENCES}

1. Akerström F, Pachón M, Puchol A, et al. Chronic right ventricular apical pacing: adverse effects and current therapeutic strategies to minimize them. Int J Cardiol 2014;173:351-60.

2. Wilkoff BL, Cook JR, Epstein AE, et al. Dual-chamber pacing or ventricular backup pacing in patients with an implantable defibrillator: the Dual Chamber and VVI Implantable Defibrillator (DAVID) Trial. JAMA 2002;288:3115-23.

3. Shimony A, Eisenberg MJ, Filion KB, et al. Beneficial effects of right ventricular non-apical vs. apical pacing: a systematic review and meta-analysis of randomized-controlled trials. Europace 2012;14:81-91.

4. Dreger $\mathrm{H}$, Maethner $\mathrm{K}$, Bondke $\mathrm{H}$, et al. Pacing-induced cardiomyopathy in patients with right ventricular stimulation for $>15$ years. Europace 2012;14:238-42.

5. Strauss DG, Selvester RH, Wagner GS. Defining left bundle branch block in the era of cardiac resynchronization therapy. Am J Cardiol 2011;107:927-34.

6. Ghani A, Delnoy PP, Ottervanger JP, et al. Assessment of left ventricular dyssynchrony in pacing-induced left bundle branch 
block compared with intrinsic left bundle branch block. Europace 2011:13:1504-7.

7. Khurshid S, Epstein AE, Verdino RJ, et al. Incidence and predictors of right ventricular pacing-induced cardiomyopathy. Heart Rhythm 2014;11:1619-25.

8. Kaye GC, Linker NJ, Marwick TH, et al. Effect of right ventricular pacing lead site on left ventricular function in patients with highgrade atrioventricular block: results of the Protect-Pace study. Eur Heart J 2015;36:856-62.

9. Udo EO, van Hemel NM, Zuithoff NP, et al. Risk of heart failure- and cardiac death gradually increases with more right ventricular pacing. Int J Cardiol 2015;185:95-100.

10. Fihn SD, Gardin JM, Abrams J, et al. ACCF/AHA/ACP/AATS/ PCNA/SCAI/STS Guideline for the diagnosis and management of patients with stable ischemic heart disease: a report of the American College of Cardiology Foundation/American Heart Association Task Force on Practice Guidelines, and the American College of Physicians, American Association for Thoracic Surgery, Preventive Cardiovascular Nurses Association, Society for Cardiovascular Angiography and Interventions, and Society of Thoracic Surgeons. $J$ Am Coll Cardiol 2012;2012:e44-e164.

11. Surawicz B, Childers R, Deal BJ, et al. AHA/ACCF/HRS recommendations for the standardization and interpretation of the electrocardiogram: part III: intraventricular conduction disturbances: a scientific statement from the American Heart Association Electrocardiography and Arrhythmias Committee, Council on Clinical Cardiology; the American College of Cardiology Foundation; and the Heart Rhythm Society: endorsed by the International Society for Computerized Electrocardiology. Circulation 2009;119:e235-e240.

12. McKee PA, Castelli WP, McNamara PM, et al. The natural history of congestive heart failure: the Framingham study. N Engl J Med 1971;285:1441-6.

13. Khurshid S, Liang JJ, Owens A, et al. Longer Paced QRS Duration is Associated With Increased Prevalence of Right Ventricular Pacing-Induced Cardiomyopathy. J Cardiovasc Electrophysiol 2016;27:1174-9.

14. Kiehl EL, Makki T, Kumar R, et al. Incidence and predictors of right ventricular pacing-induced cardiomyopathy in patients with complete atrioventricular block and preserved left ventricular systolic function. Heart Rhythm 2016:13:2272-8.

15. Stambler BS, Ellenbogen $\mathrm{K}$, Zhang $\mathrm{X}$, et al. Right ventricular outflow versus apical pacing in pacemaker patients with congestive heart failure and atrial fibrillation. $J$ Cardiovasc Electrophysiol 2003;14:1180-6.

16. Chan NY, Yuen $\mathrm{HC}$, Choy $\mathrm{CC}$, et al. Left ventricular volumes and systolic function after long-term right ventricular pacing may be predicted by paced QRS duration, but not pacing site. Heart Lung Circ 2014;23:43-8.

17. Chen S, Yin Y, Lan X, et al. Paced QRS duration as a predictor for clinical heart failure events during right ventricular apical pacing in patients with idiopathic complete atrioventricular block: results from an observational cohort study (PREDICT-HF). Eur J Heart Fail 2013;15:352-9.

18. Pan W, Su Y, Gong X, et al. Value of the paced QRS duration. J Card Fail 2009;15:347-52.

19. Pap R, Fürge P, Bencsik G, et al. Native QRS complex duration predicts paced QRS width in patients with normal left ventricular function and right ventricular pacing for atrioventricular block. $J$ Electrocardiol 2007:40:360-4.

20. Pan W, Su Y, Sun A, et al. Native and paced QRS duration in right ventricular apex paced patients. J Card Fail 2010;16:239-43.

21. Miyoshi F, Kobayashi $\mathrm{Y}$, Itou $\mathrm{H}$, et al. Prolonged paced QRS duration as a predictor for congestive heart failure in patients with right ventricular apical pacing. Pacing Clin Electrophysiol 2005;28:1182-8.

22. Fonseca $\mathrm{C}$, Morais $\mathrm{H}$, Mota $\mathrm{T}$, et al. The diagnosis of heart failure in primary care: value of symptoms and signs. Eur $\mathrm{J}$ Heart Fail 2004:6:795-800.

23. Thosani AJ, Liu E, Shaw G, et al. Rapid reversal of right ventricular pacing-induced cardiomyopathy by His bundle pacing. HeartRhythm Case Rep 2017;3:189-91.

24. Aijiola OA, Upadhyay GA, Macias C, et al. Permanent His-bundle pacing for cardiac resynchronization therapy: Initial feasibility study in lieu of left ventricular lead. Heart Rhythm 2017;14:1353-61.

25. Shah KS, Xu H, Matsouaka RA, et al. Heart Failure With Preserved, Borderline, and Reduced Ejection Fraction: 5-Year Outcomes. J Am Coll Cardiol 2017:2476-86. 